University of South Carolina

Scholar Commons

2-15-2008

\title{
Current-Voltage Characteristics of Semiconductor/Ferromagnet Junctions in the Spin-Blockade Regime
}

Yuriy V. Pershin Dr

University of South Carolina - Columbia, pershin@physics.sc.edu

Massimiliano Di Ventra

Follow this and additional works at: https://scholarcommons.sc.edu/phys_facpub

Part of the Physics Commons

Publication Info

Published in Physical Review B, ed. Gene D. Sprouse, Volume 77, Issue 7, 2008, pages

073301-1-073301-4.

Pershin, Y. V., \& Di Ventra, M. (2008). Current-voltage characteristics of semiconductor/ferromagnet junctions in the spin-blockade regime. Physical Review B, 77(7), 073301-1 - 073301-4. DOI: 10.1103/ PhysRevB.77.073301

(c) Physical Review B, 2008, American Physical Society

This Article is brought to you by the Physics and Astronomy, Department of at Scholar Commons. It has been accepted for inclusion in Faculty Publications by an authorized administrator of Scholar Commons. For more information, please contact digres@mailbox.sc.edu. 


\title{
Current-voltage characteristics of semiconductor/ferromagnet junctions in the spin-blockade regime
}

\begin{abstract}
Yuriy V. Pershin* and Massimiliano Di Ventra ${ }^{\dagger}$
Department of Physics, University of California, San Diego, La Jolla, California 92093-0319, USA

(Received 18 September 2007; revised manuscript received 11 December 2007; published 7 February 2008)

It was recently predicted [Phys. Rev. B 75, 193301 (2007)] that spin blockade may develop at nonmagnetic semiconductor/perfect ferromagnet junctions when the electron flow is directed from the semiconductor into the ferromagnet. Here we consider current-voltage characteristics of such junctions. By taking into account the contact resistance, we demonstrate a current stabilization effect: by increasing the applied voltage, the current density through the junction saturates at a specific value. The transient behavior of the current density is also investigated. We show that an abrupt change in the applied voltage is accompanied by a spike in the current density. It is anticipated that this is a common dynamical behavior of the current density in structures with conductivity depending on the level of spin polarization.
\end{abstract}

DOI: 10.1103/PhysRevB.77.073301

PACS number(s): 73.23.Hk, 72.25.Dc, 72.25.Mk

There is currently a great deal of interest in spindependent transport phenomena in semiconductors/ ferromagnet junctions. ${ }^{1-13}$ In large part, this interest is motivated by the goal to exploit these phenomena in new technologies, such as spintronics and quantum computation. ${ }^{1}$ Recently, some attention has been focused on the problem of the extraction of spin-polarized electrons from the semiconductor to the ferromagnet. ${ }^{2,7-13}$ The importance of spin extraction was already realized in early studies of spinpolarized transport in magnetic semiconductors. ${ }^{7,8}$ However, despite the apparent similarity of spin extraction with spin injection, the former shows unique features.

We have recently predicted ${ }^{2}$ that the spin extraction process at nonmagnetic semiconductor/perfect ferromagnet junctions can be limited by spin blockade. The physical mechanism for spin blockade can be understood as follows. Let us consider a current flowing through the junction in such a way that the electron motion is directed from the semiconductor into the ferromagnet. The perfect ferromagnet accepts electrons of only one spin direction (majority-spin electrons). Without possibility to enter the ferromagnet, the minority-spin electrons gather near the junction, forming a cloud. With increased current, this minority-spin cloud increases, and at a given critical current $j_{c}$ the amount of majority-spin electrons near the junction becomes insufficient to sustain a further current increase. Therefore, the majority-spin electron current through the junction becomes blocked by minority-spin electrons accumulated near the boundary. ${ }^{2}$ This kind of spin blockade is collective and based on Coulomb interaction, while in quantum dots the spin blockade occurs on the single electron level and is due to the Pauli exclusion principle, ${ }^{14}$ which prevents current passing through two quantum dots in series, if the spin directions of electrons in these dots are the same. ${ }^{14}$

In this Brief Report we explore experimentally verifiable consequences of the spin-blockade phenomenon. In particular, we study the current-voltage characteristics of junctions where this effect occurs. We do so by considering the conductivity of each of its components (semiconductor, ferromagnet, and their contact). We show that the current flowing in a circuit involving a semiconductor/perfect ferromagnet interface in the spin-blockade regime saturates with increasing applied voltage. Therefore, such an interface can be potentially used as a spin-based current stabilizer. We also show that in structures with a semiconducting region longer than the spin-diffusion length, the current density saturates to the critical current density $j_{c}$ found in Ref. 2. Instead, in junctions with the semiconductor region shorter than the spin-diffusion length, the asymptotic current value may be different from $j_{c}$ depending on how the semiconductor is connected from the opposite side of the junction. In particular, if this second contact is a good contact with a normal metal, then the asymptotic current value is higher than $j_{c}$. We note that the current stabilization effect is possible only in the junctions with $100 \%$ spin polarization ferromagnets. ${ }^{2} \mathrm{We}$ also consider transient processes, which, due to the finite response time of the spin polarization to the applied voltage, limit the speed of operation of such devices.

The circuit we have in mind is shown schematically in the Fig. 1. We consider a voltage source (battery) connected to the semiconductor and ferromagnet regions of the junction. Assuming that the ferromagnet is a good conductor we can neglect the voltage drop across it. We also assume a good contact of the voltage source with the semiconductor (Ohmic or nonlinear contact at this junction can be easily incorporated into our model). Therefore, there are two components of the total circuit where the voltage mainly drops: the semiconductor region and its contact with the ferromagnet. We can then write the total applied voltage $V$ as $V=V_{s}+V_{c}$, where $V_{s}$ and $V_{c}$ are voltage drops across the semiconductor region and the contact, respectively. In our model, we consider a perfect ferromagnet, such as a half-metal ferromag-

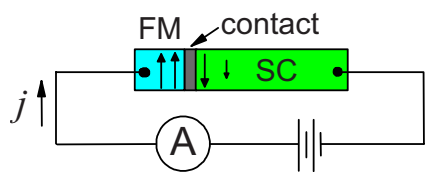

FIG. 1. (Color online) Schematic drawing of the circuit: the outflow of spin-up electrons from the semiconductor (SC) into ferromagnet (FM) leaves a cloud of spin-down electrons in the semiconductor near the contact. 
net. While both spin-up and spin-down electrons are injected from the battery into the semiconductor, only, say, spin-up electrons are extracted from the semiconductor into the ferromagnet.

Spin and charge transport of a nondegenerate electron gas in the semiconductor can be conveniently described within the drift-diffusion approximation.,17 Our goal is to obtain analytical expressions for the current so, as in Ref. 2, we neglect charge redistribution effects near the junction, such as Schottky barriers. ${ }^{4}$ These effects can be included into our scheme through a nonlinear contact resistance, and we do not expect them to modify the qualitative behavior of the results we discuss here. In the semiconductor region we can then write

$$
j=\sigma E=e N_{0} \mu \frac{V_{s}}{L} \equiv \frac{V_{s}}{\rho_{s} L},
$$

where $j$ is the current density, $\sigma$ is the conductivity, $E$ is the electric field, $\mu$ is the mobility defined via $\vec{v}_{d r i f t}=\mu \vec{E}, L$ is the length of the semiconductor, $\rho_{s}$ is the semiconductor resistivity, $-e$ is the electron charge, and $N_{0}$ is the electron density in the semiconductor. Next, we consider the voltage drop across the contact. The conductivity of the contact is proportional to the density of majority spin electrons in the semiconductor near the contact, $n_{\uparrow}(0)$. Therefore, assuming a linear relationship between the current and voltage drop across the contact at a fixed spin-up density, $n_{\uparrow}(0)$, we write

$$
j=\frac{2 n_{\uparrow}(0)}{N_{0}} \frac{V_{c}}{\rho_{c}^{0}},
$$

where $\rho_{c}^{0}$ is the steady-state contact resistivity at $V \rightarrow 0$ [when $\left.n_{\uparrow}(0)=N_{0} / 2\right]$. Combining Eqs. (1) and (2) we get

$$
V=V_{s}+V_{c}=\left[\rho_{s} L+\rho_{c}^{0} \frac{N_{0}}{2 n_{\uparrow}(0)}\right] j .
$$

Equation (3), which couples $V$ and $j$, must be supplemented by the system of drift-diffusion equations for the semiconductor region whose solution gives $n_{\uparrow}(0)$. This system of equations consists of the continuity equations for spin-up and spin-down electrons, and the equations for the two spin currents

$$
\begin{gathered}
e \frac{\partial n_{\uparrow(\downarrow)}}{\partial t}=\operatorname{div} \vec{j}_{\uparrow(\downarrow)}+\frac{e}{2 \tau_{s f}}\left(n_{\downarrow(\uparrow)}-n_{\uparrow(\downarrow)}\right), \\
\vec{j}_{\uparrow(\downarrow)}=\sigma \vec{E}+e D \nabla n_{\uparrow(\downarrow)} .
\end{gathered}
$$

Here, $D$ is the diffusion coefficient and $\tau_{s f}$ is the spin relaxation time. It is assumed that the total electron density in the semiconductor is constant, i.e., $n_{\uparrow}(x)+n_{\downarrow}(x)=N_{0}$. Correspondingly, the electric field is homogeneous and coupled to the total current density as $j=e \mu N_{0} E_{0}$. The boundary conditions are $j_{\uparrow}(0)=j, j_{\downarrow}(0)=0$, and $n_{\uparrow}(L)=n_{\downarrow}(L)=N_{0} / 2$.

In the following, we will consider separately the two cases of long $\left(L \gg l_{s}\right)$ and short $\left(L \lesssim l_{s}\right)$ semiconductor regions, with $l_{s}$ the spin-diffusion length defined below.

(i) $L \gg l_{s}$. In this limit, a steady-state solution of Eqs. (4)

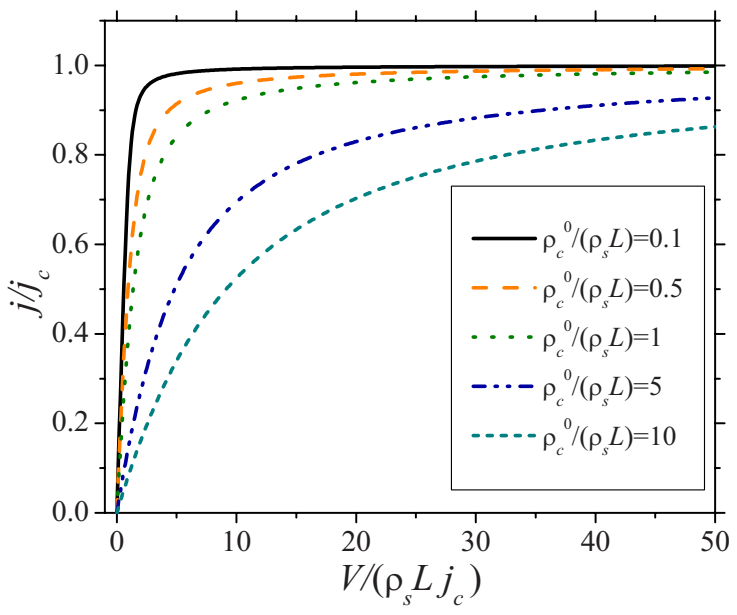

FIG. 2. (Color online) Current-voltage characteristics of the system calculated for several values of the ratio between the contact resistance and the semiconductor resistance $\rho_{c}^{0} /\left(\rho_{s} L\right)$ (see text for details).

and (5) is known. ${ }^{2}$ The spin densities decay exponentially from the junction to their bulk values of $N_{0} / 2$. The decay occurs on the length scale of the up-stream spin-diffusion length ${ }^{2,17} l_{s}=2 D /\left(\mu E_{0}+\sqrt{\mu^{2} E_{0}^{2}+4 D / \tau_{s f}}\right)$. The spin-up density at the junction is ${ }^{2}$

$$
n_{\uparrow}(x=0)=\frac{N_{0}}{2}-\frac{N_{0}}{\sqrt{1+4 \frac{D}{\tau_{s f} \mu^{2} E_{0}^{2}}}-1} .
$$

Substituting Eq. (6) into Eq. (3) and introducing the dimensionless current density $\tilde{j}=j / j_{c}$, where

$$
j_{c}=e N_{0} \sqrt{\frac{D}{2 \tau_{s f}}}
$$

is the critical current density, ${ }^{2}$ we get a closed equation coupling current density and voltage as follows:

$$
\frac{V}{\rho_{s} L j_{c}}=\left(1+\frac{\rho_{c}^{0}}{\rho_{s} L} \frac{1}{1-\frac{2}{\sqrt{1+\frac{8}{\tilde{j}^{2}}}-1}}\right) \tilde{j} .
$$

Figure 2 shows solutions of Eq. (8) at different values of the ratio of the contact resistance to the resistance of the semiconductor region. All curves saturate at $j / j_{c}=1$ with increasing voltage. The saturation occurs faster in systems having smaller contact resistance. In Fig. 3, we plot the corresponding spin-up density $n_{\uparrow}(0)$. It follows from Figs. 2 and 3 that, for the selected values of parameters, the current density $j$ is quite close to the critical current density $j_{c}$ at voltages for which $2 n_{\uparrow}(0) / N_{0} \sim 10^{-2}$. For current stabilization applications, by specifying the maximum desired deviation of $j$ from $j_{c}$, one can obtain the minimal voltage $V_{\min }$ required for that deviation using Eq. (8). ${ }^{15}$ 


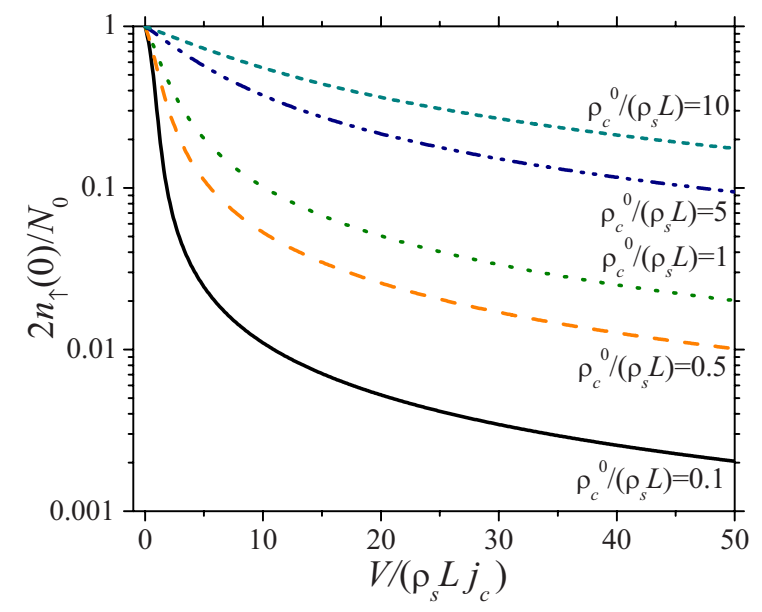

FIG. 3. (Color online) Spin-up density at the junction monotonically decreases with increase of the applied voltage $V$.

(ii) $L \leqq l_{s}$. In this limit, Eqs. (4) and (5), supplemented by Eq. (3), were solved numerically using the ScharfetterGummel discretization scheme ${ }^{16}$ which gives reliable numerical results. ${ }^{7}$ Starting with unpolarized electrons in the semiconductor, we have iterated at each time step Eqs. (4) and (5) with the constrain imposed by Eq. (3).

In this regime, the current-voltage characteristics have a similar saturation behavior as in the case $L \gg l_{s}$. However, the asymptotic values of the current density $(t \rightarrow \infty, V \rightarrow \infty)$ are higher than $j_{c}$ given by Eq. (7) (see Fig. 4). This is due to the boundary conditions $n_{\uparrow}(L)=n_{\downarrow}(L)=N_{0} / 2$. Such boundary conditions describe a perfect contact of the semiconductor with a large reservoir of spin-unpolarized electrons. These

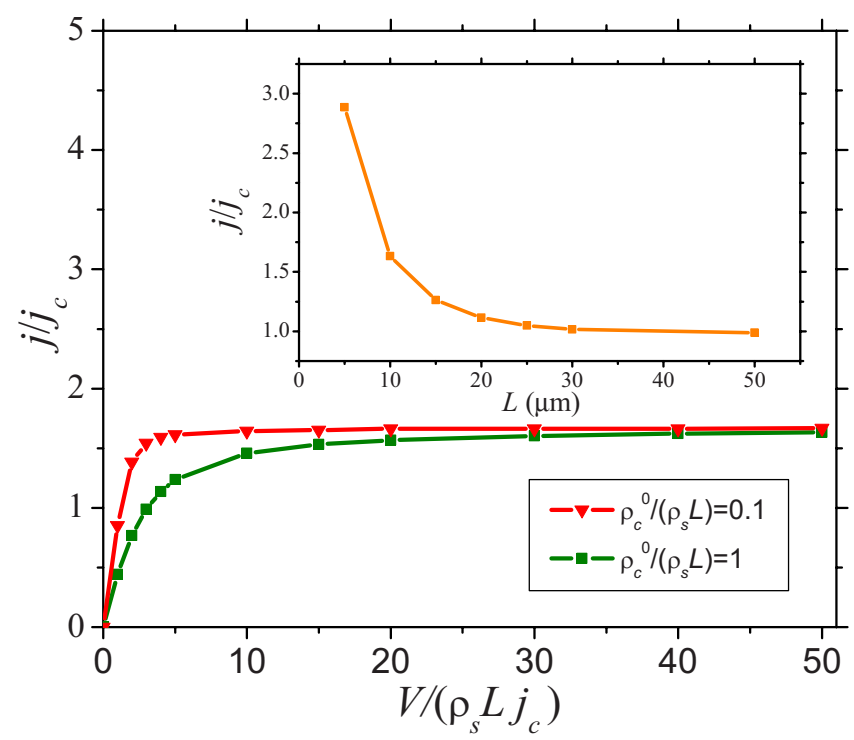

FIG. 4. (Color online) Current-voltage characteristics of a junction with $L=10 \mu \mathrm{m}$ showing the current density saturation at $j$ $>j_{c}$. Inset: steady-state current density as a function of $L$ at the fixed value of $V /\left(\rho_{s} L j_{c}\right)=50$. These plots were obtained using parameter values $D=220 \mathrm{~cm}^{2} / \mathrm{s}, \quad \mu=8500 \mathrm{~cm}^{2} /(\mathrm{V} \mathrm{s}), \quad N_{0}=5$ $\times 10^{15} \mathrm{~cm}^{-3}$, and $\tau_{s f}=10 \mathrm{~ns}$. The corresponding critical current density $j_{c}$ [calculated using Eq. (7)] is $8.4 \times 10^{-7} \mathrm{~A} / \mathrm{cm}^{2}$.

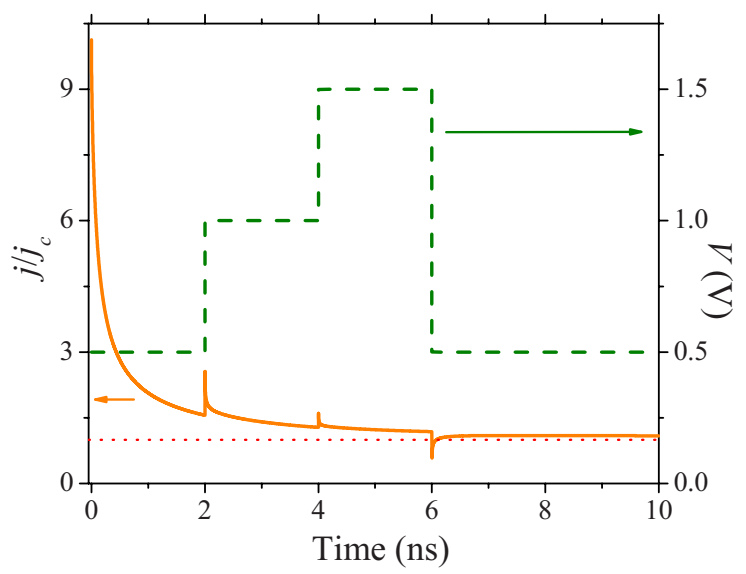

FIG. 5. (Color online) Transient current (solid line) excited by application of step voltages (dashed line). The dotted horizontal line, corresponding to $j=j_{c}$, is a guide for the eyes. Here we used $L=20 \mu \mathrm{m}$ and $\rho_{c}^{0} /\left(\rho_{s} L\right)=1$. The rest of the parameters are shown in Fig. 4.

spin-unpolarized electrons facilitate diffusion of electrons from the contact region, reducing the level of spin polarization near the contact and thus increasing the current density at which spin blockade occurs. We plot the current density as a function of $L$ in the inset of Fig. 4. For the selected set of parameters, the current density starts to deviate noticeably from $j_{c}$ in structures with $L \lesssim 20 \mu \mathrm{m}$.

Moreover, in view of potential applications, it is important to know the transient behavior of the current density. To do this, we consider stepwise voltage changes as shown in Fig. 5. This illustrative shape of $V$ was selected to show the response to both positive and negative voltage increments. The resultant current density depicted in Fig. 5 exhibits spikes at each change in $V$. The main change in current density occurs during the first several hundreds of picoseconds after the voltage is applied.

Physically, when voltage changes, the electron spin polarization adjusts to a new value of the bias and this adjustment process takes some time during which the spikes are observed. Within a short initial time period immediately after the voltage change, the spin polarization near the contact keeps its memory on a previous voltage value. For example, immediately after an increase of $V, n_{\uparrow}(x=0, t)$ still corresponds to a previous value of bias which, according to Eqs. (1) and (3), gives higher contact conductivity than its equilibrium value at the new voltage. Therefore, we observe an abrupt current increase on the $I-V$ curve. As time passes, $n_{\uparrow}(x=0, t)$ relaxes to its new equilibrium value corresponding to a smaller contact conductivity, which manifested as decrease in current. That is how positive current spikes are formed. Similarly, a stepwise decrease of $V$ results in a negative spike. We finally note that current density spikes cannot be fitted by a single exponent.

Let us also estimate the critical electric field $E_{c}$ in the semiconductor corresponding to the critical current $j_{c}$. From Eqs. (1) and (7) we easily obtain 


$$
E_{c}=\sqrt{\frac{D}{2 \tau_{s f} \mu^{2}}} .
$$

It is interesting to note that $E_{c}$ does not directly depend on the electron density. Substituting GaAs parameters $D$ $=220 \mathrm{~cm}^{2} / \mathrm{s}, \mu=8500 \mathrm{~cm}^{2} /(\mathrm{V} \mathrm{s})$, and $\tau_{s f}=10 \mathrm{~ns}$ we find $E_{c}$ $\simeq 12 \mathrm{~V} / \mathrm{cm}$. This is a very weak electric field, so that the use of field-independent spin relaxation time in Eq. (4) is well justified.

In conclusion, we have shown in this Brief Report that there is a wealth of interesting physics behind the process of spin extraction from semiconductors. We have reported current-voltage characteristics of semiconductor/perfect ferromagnet junctions in the spin-blockade regime using a driftdiffusion approximation. We have demonstrated that the spin-blockade phenomenon we have predicted $^{2}$ leads to a current stabilization effect, which can be directly measured. We have also predicted transient spikes in current density. Our results are important to understand the process of spin extraction, and may find application in the design of spinbased electronic devices. We thus hope our work will motivate experiments in this direction.

We acknowledge discussions with G. Vignale. This work is partly supported by the NSF Grant No. DMR-0133075. *pershin@physics.ucsd.edu

†diventra@physics.ucsd.edu

${ }^{1}$ I. Žutić, J. Fabian, and S. Das Sarma, Rev. Mod. Phys. 76, 323 (2004).

${ }^{2}$ Y. V. Pershin and M. Di Ventra, Phys. Rev. B 75, 193301 (2007).

${ }^{3}$ E. I. Rashba, Eur. Phys. J. B 29, 513 (2002).

${ }^{4}$ J. D. Albrecht and D. L. Smith, Phys. Rev. B 68, 035340 (2003); 66, 113303 (2002).

${ }^{5}$ M. Shen, S. Saikin, and M.-C. Cheng, J. Appl. Phys. 96, 4319 (2004).

${ }^{6}$ Y. Y. Wang and M. W. Wu, Phys. Rev. B 72, 153301 (2005).

${ }^{7}$ I. Žutić, J. Fabian, and S. Das Sarma, Phys. Rev. Lett. 88, 066603 (2002).

${ }^{8}$ J. Fabian, I. Žutić, and S. Das Sarma, Phys. Rev. B 66, 165301 (2002).

${ }^{9}$ R. K. Kawakami, Y. Kato, M. Hanson, I. Malajovich, J. M. Stephens, E. Johnston-Halperin, G. Salis, A. C. Gossard, and D.
D. Awschalom, Science 294, 131 (2001).

${ }^{10}$ R. J. Epstein, I. Malajovich, R. K. Kawakami, Y. Chye, M. Hanson, P. M. Petroff, A. C. Gossard, and D. D. Awschalom, Phys. Rev. B 65, 121202(R) (2002).

${ }^{11}$ J. Stephens, J. Berezovsky, J. P. McGuire, L. J. Sham, A. C. Gossard, and D. D. Awschalom, Phys. Rev. Lett. 93, 097602 (2004).

${ }^{12}$ S. A. Crooker, M. Furis, X. Lou, C. Adelmann, D. L. Smith, C. J. Palmstrom, and P. A. Crowell, Science 309, 2191 (2005).

${ }^{13}$ H. Dery and L. J. Sham, Phys. Rev. Lett. 98, 046602 (2007).

${ }^{14}$ K. Ono, D. G. Austing, Y. Tokura, and S. Tarucha, Science 297, 1313 (2002).

${ }^{15}$ We note that $V_{\min }$ is linear in $\rho_{c}$.

${ }^{16}$ D. L. Scharfetter and H. K. Gummel, IEEE Trans. Electron Devices ED-16, 64 (1969).

${ }^{17}$ Z. G. Yu and M. E. Flatté, Phys. Rev. B 66, 201202(R) (2002). 